
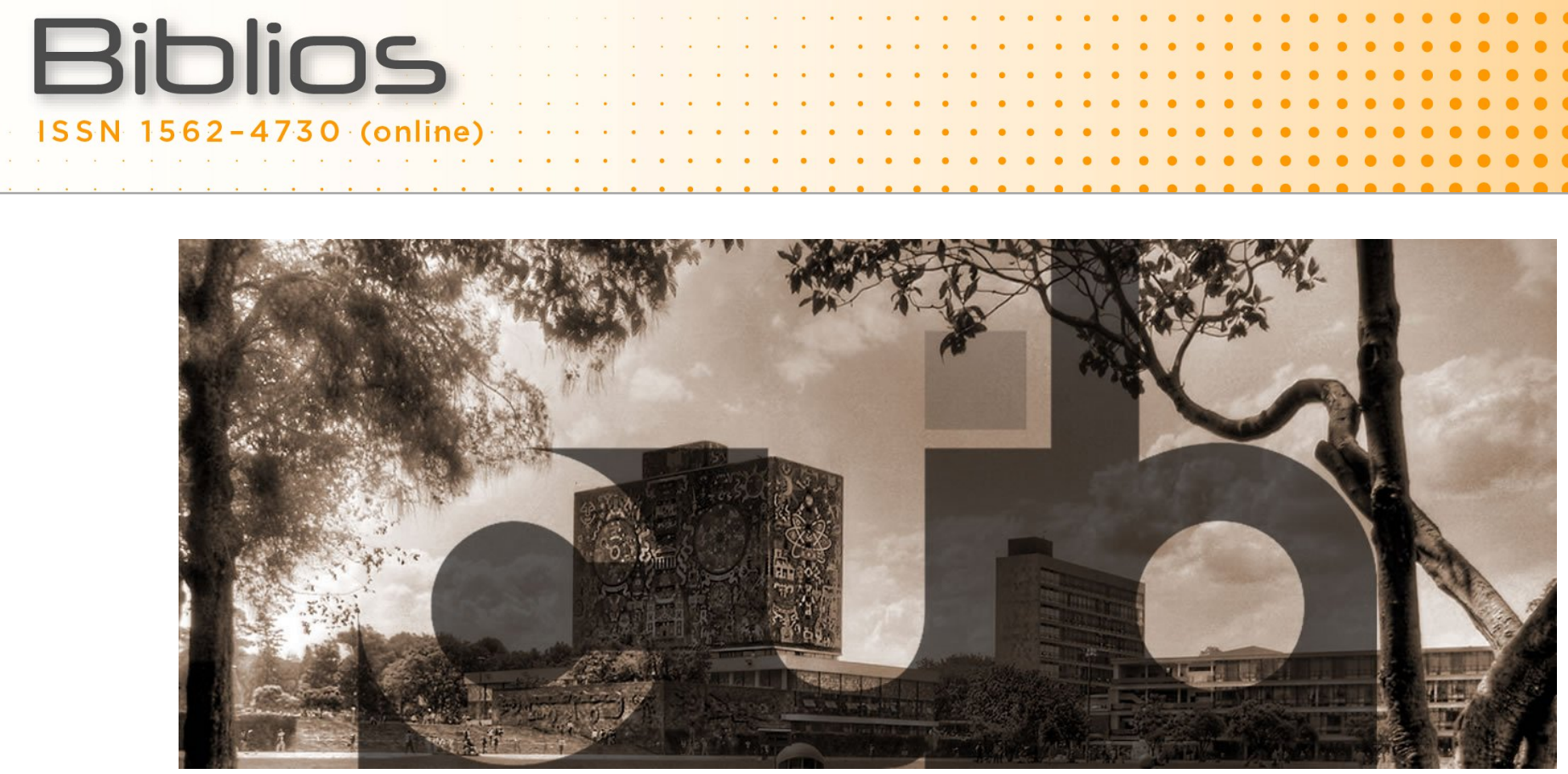

\title{
EI CUIB: Un foco de motivación para la investigación sobre Bibliotecología y Ciencia de la Información en América
}

\section{Latina}

\section{INTERVIEW}

\section{Resumo}

Entrevista a Filiberto Felipe Martínez Arellano (México) investigador del Centro Universitario de Investigaciones Bibliotecológicas (CUIB) en el marco del treinta aniversario de este centro académico.

\section{Palavras-chave}

Filiberto Felipe Martínez Arellano; Centro Universitario de Investigaciones Bibliotecologicas; CUIB; Centros de investigación; Formación profesional; Entrevistas

The CUIB: A source of motivation for research on librarianship and information science in Latin America

Abstract Title

Interview with Filiberto Felipe Martínez Arellano (Mexico) researcher of the Centro Universitario de Investigaciones Bibliotecologicas (CUIB) in the context of the thirtieth anniversary of this academic center.

Keywords Title

Filiberto Felipe Martínez Arellano; Centro Universitario de Investigaciones Bibliotecologicas; CUIB; Research centers; Vocational training; Interviews 
El 14 de diciembre de 1981 fue creado en la Universidad Nacional Autónoma de México (UNAM), el Centro Universitario de Investigaciones Bibliotecológicas (CUIB), con la finalidad de llevar a cabo investigaciones teóricas y aplicadas sobre los fenómenos relacionados con la información registrada en impresos y otros medios: sus formas de generación, su selección y adquisición, su organización y representación, así como sobre los problemas concernientes con los medios, procesos y tecnologías utilizados para el almacenamiento, recuperación y distribución de la información.

A lo largo de tres décadas esta organización ha acumulado una rica experiencia de trabajo, sobre la cual conversamos con Filiberto Felipe Martínez Arellano ex director y uno de los principales investigadores de este centro.

\section{¿Cómo resumiría las tres décadas de labor del CUIB?}

Martínez: A Creo que uno de los hitos para la disciplina fue precisamente el surgimiento del CUIB, el cual permitió tener un espacio para la investigación paralelo a la práctica profesional. Ese fue un punto de partida muy importante que tuvo impacto para nuestra disciplina.

Posteriormente otro gran cambio del CUIB fue abrir un espacio para la investigación en la universidad y que otras disciplinas se dieran cuenta de que somos capaces no únicamente de llevar a cabo práctica profesional, sino también llevar a cabo investigación, con métodos, con técnicas de investigación, totalmente universales, globales, que se utilizan en cualquier otra disciplina. Eso obviamente trajo una serie de demandas, la demanda principal es que otras disciplinas, de cualquier índole, no necesariamente de índole humanística, sino en las ciencias puras, decían que sus investigadores eran doctores y que hacer investigación, decían, se aprende a hacerla en el doctorado, lo cual nos llevó a que nuestro personal tuviese que alcanzar los estándares, las mismas condiciones con la que trabaja cualquier otro instituto de cualquier otra disciplina dentro de la universidad.

Trajo sus beneficios, poder decirles a los investigadores de otras disciplinas: somos pares, tú tienes investigadores doctores, yo también tengo investigadores doctores, tú perteneces al Sistema Nacional de Investigadores - SNI, yo también pertenezco, tú tienes investigadores que participan como árbitros en revistas de carácter internacional, yo también tengo.

En sí, creo que las aportaciones, los cambios sustanciales del CUIB en estos treinta años, ha sido un fortalecimiento de la disciplina, y una credibilidad de que en bibliotecología también se puede hacer investigación; que la bibliotecología no se reduce únicamente a la práctica profesional, que la práctica profesional es importante y que esta siempre debe ser acompañada por una reflexión epistemológica, sistemática, de lo que hacemos cada día. Ese ha sido el gran cambio en el CUIB.

\section{¿Qué representa el CUIB en el desarrollo de la Bibliotecología y la Ciencia de la Información en América Latina?}

Martínez: El CUIB fue un foco de motivación para la investigación en América Latina. Antes de la existencia del CUIB la investigación se hacía conjuntamente con la práctica profesional; los bibliotecarios, los bibliotecólogos en la práctica profesional, adicionalmente llevaban tareas de investigación. Sin embargo, con la creación del CUIB se empezaron a formar otros centros, otras unidades de investigación en distintos países de América Latina; y empezó a motivar también a que en las escuelas de bibliotecología y ciencias de la información se empezara a llevar a cabo proyectos de investigación. Y ahora vemos que existen proyectos de investigación en diferentes escuelas, existen grupos de investigación, existen aportes. Indirectamente el CUIB motivó la investigación en esta región.

La generación de la literatura en español hacia América Latina; anteriormente a la creación del CUIB, toda la literatura científica de calidad se publicaba únicamente en inglés, en revistas publicadas en el extranjero. A raíz de la generación de literatura en español, los investigadores, los profesores de América Latina, tuvieron un medio para comunicar los resultados de sus investigaciones. Y surge una revista que es Investigación Bibliotecológica, la cual 
ha alcanzado un índice de calidad, de excelencia; está indizada en el Social Science Citation Index, un índice de carácter internacional, y por eso mismo existe una gran demanda para publicar en esta revista.

Y otro aspecto importante que me gustaría señalar es que se convirtió en el centro de discusión, por excelencia, para discutir y analizar el problema de la investigación bibliotecológica, problemas de la región, la bibliotecología y las ciencias de la información en América Latina, con los encuentros, con las actividades que se llevan a cabo. Es el centro por excelencia de reuniones donde los investigadores, los profesores de América Latina acudimos a discutir la base de diversas temáticas. Un ejemplo de ello es el encuentro que celebramos anualmente sobre investigación bibliotecológica, el cual ya va en el veintiocho encuentro de investigación bibliotecológica, en el cual han participado a lo largo de todos estos, colegas de América Latina, que nos hemos retroalimentado mutuamente. Así mismo el encuentro de catalogación que celebramos anualmente, han participado gentes de América Latina. Para América Latina también ha sido importante.

Por último, el programa de pasantías. El Programa de Pasantías que ofrece el CUIB donde colegas de América Latina pueden estar desde una semana hasta seis meses trabajando proyectos de investigación continúa con algún investigador del centro. Hemos tenido a lo largo de estos treinta un sin número de investigadores, de profesionales de la bibliotecología que han estado en el CUIB trabajando en algún proyecto, retro alimentándonos mutuamente, trabajando de manera sistemática.

Para América Latina entonces el CUIB ha sido un foco de generación de investigaciones, de motivación de las investigaciones de nuestra región, de problemas de nuestra región. Ha sido un centro donde se genera una revista donde participan los bibliotecólogos, los investigadores, los profesionales de América Latina. Un centro de discusión académica, de diversos problemas en América Latina, un centro de información en investigación y retroalimentación mutua, donde han acudido muchos colegas de América Latina.Que representa el CUIB en el desarrollo de la Bibliotecología y la Ciencia de la Información en América Latina?

\section{¿Cuales son los proyectos futuros del CUIB?}

Martínez: Yo vería como ex-director del CUIB dos grandes proyectos. Uno que inicié, que es transformarnos dentro de la estructura de la universidad, de centro a instituto. En nuestra universidad cualquier entidad, cualquier organización nace primeramente como un centro. A medida como transcurre el tiempo y va demostrando que ha alcanzado una madurez académica, mostrando diversas evidencias, con el número de investigadores, el grado obtenido por sus investigadores, el número de publicaciones de sus investigadores, el impacto de estas publicaciones de los investigadores en el ámbito internacional, en este caso América Latina, la formación de recursos, que en este momento tenemos un doctorado también, creo que en este momento todos elementos nos permiten aspirar a un instituto. Un instituto que dentro de la estructura académica, pues tiene ciertos privilegios. Los privilegios son demostrar a la comunidad académica que hemos madurado, el poder participar en la toma de decisiones de la universidad. Participar en la toma de decisiones de una universidad es únicamente derecho de un instituto que ha demostrado que académicamente ha alcanzado la madurez. Estamos en el camino de esto y esperamos que en el futuro alcancemos el rango de instituto.

Por otro lado, otro de los retos del centro es el crecimiento cuantitativo. En este momento somos veinticinco investigadores y creo que hacia futuro el CUIB debe de crecer cuantitativamente en el número de investigadores, para abordar otra serie de temáticas que en este momento no se abordan. No se abordan no porque no estemos conscientes de ello, sino porque los recursos que tenemos en el centro son insuficientes.

Y otro aspecto importante hacia el futuro es tener una proyección de carácter internacional. Esto significa que tenemos que interactuar con los pares de otros países, no únicamente de América Latina, sino a nivel global. Creo que tenemos la madurez académica, creo que tenemos los elementos ahora si para interactuar de par a par con centros de investigación en los países en vías de desarrollo. Desde mi perspectiva ese tendría que ser el futuro del CUIB.

Un crecimiento a otro estatus dentro de nuestra misma institución a un crecimiento cuantitativo que nos permita abordar mayores temáticas de la investigación y a una proyección de carácter internacional que demuestre a nivel global que el CUIB ha alcanzado una madurez académica, la cual tiene. Y como evidencia es el número de 
investigadores, el número de proyectos que se realizan, el número de gentes formadas. Como mencionaba anteriormente el CUIB ha promovido el establecimiento de un doctorado a partir de hace diez años, uno de los primeros doctorados que se ofrecen en América Latina en idioma español, no hay otra institución que esté ofreciendo un doctorado en español, a excepción de Cuba, los otros doctorados están ofrecidos en portugués.

\section{¿Qué reflexiones finales compartiría tras su labor en el CUIB?}

Martínez: Creo que en América Latina hemos trabajado por muchos años para el desarrollo de la bibliotecología, desde las diversas trincheras, desde los diversos lugares, desde la práctica profesional, desde las escuelas de bibliotecología, en donde llevamos a cabo investigación, docencia.

Creo que la bibliotecología en América Latina tiene un gran futuro y un gran potencial. A lo largo de mi carrera y desarrollo profesional he sido testigo el gran avance que ha tenido la bibliotecología latinoamericana. En los diversos lugares de América Latina estamos desarrollando proyectos, estamos desarrollando investigaciones, estamos formando gente y contribuyendo al desarrollo de la bibliotecología en nuestra región.

Un saludo para todos ustedes.

\section{Producción}

Transcripción y edición: Julio Santillán-Aldana

Agradecimento:

Pontificia Universidad Católica del Perú. Especialidad de Ciencia de la Información.

IFLA Latin America and the Caribbean Section.

\section{(cc) BY-NC-ND}

This work is licensed under a Creative Commons Attribution-Noncommercial-No Derivative Works 3.0 United States License.

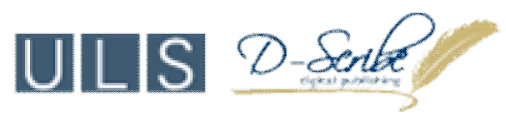

This journal is published by the University Library System of the University of Pittsburgh as part of its $\underline{D-S c r i b e}$ Digital Publishing Program and is cosponsored by the University of Pittsburgh Press. 